\title{
Inthaltswerz̧eidnis.
}

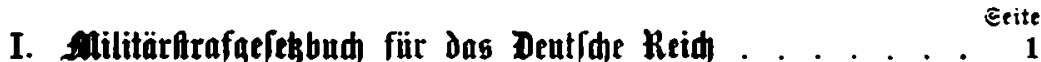

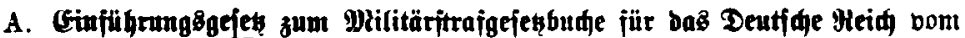
20. Juni 1872 . . . . . . . . . . . . . . . . . . . 1

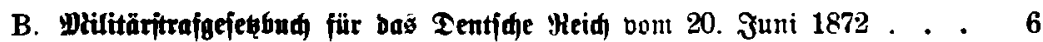

Einleitende Beftimmungen . . . . . . . . . . . . . . . . . . 6

Eriter Teil: Bon ber bejtraing im allgemeinen.

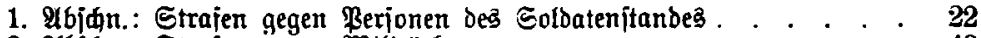

2. Mbjan.: Strafen gegen $\mathfrak{M}$ iflitärbeante . . . . . . . . . . . 49

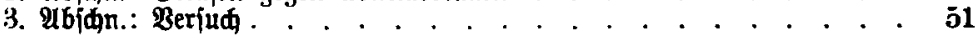

4. Abiqn.: Teilnahme. . . . . . . . . . . . . . 52

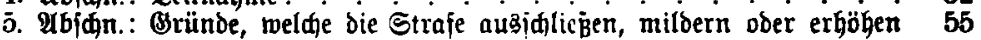

3weiter Teil: Bon ben einzelnen Berbreqen und Bergehen und beren 8 eitrafung.

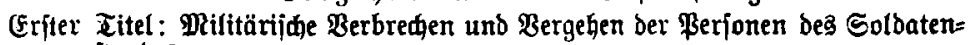
ftandes . . . . . . . . . . . . . . . . . . . . 67

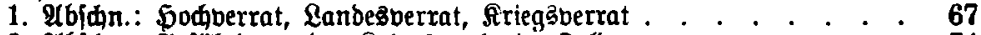

2. Ubidn.: Befähroung ber Sriegamadit im Felbe . . . . . . . . 74

3. $\mathfrak{A b i a n}$ : Inerlaubte Entfernung und Fahnenfludt . . . . . . . 76

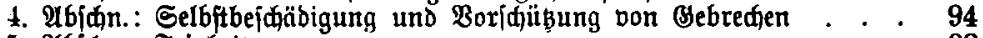

כ. Mbidn.: Freigheit . . . . . . . . . . . . 98

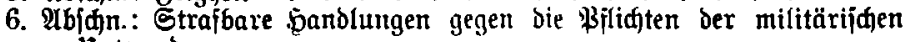
unteroronung . . . . . . . . . 103

7. Mbjan.: Mriß̧braud) ber Dienitgewalt . . . . . . . . . . . 153

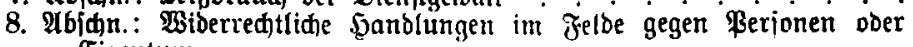
厄igentum . . . . . 174

9. थbfan.: Undere viberredtitidie bandiungen gegen bas (Eigentum . 184

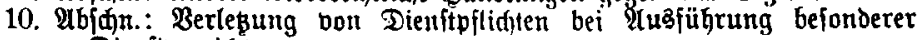
Dienftberridtungen. . . . . . . . . 188

11. Abiqn.: Sonjtige Sandlungen gegen die militärtique sronung. . 202

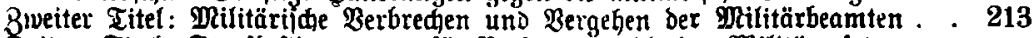

Dritter Titel: Strafbeftimmungen für ßerionen, welthe ben Militärgéfegen nur in §riegszeiten unterworjen jind . . . . . . . 214

Bierter Titel: Zufaßbeitimımungen für bie Miarine: $: . .+. .+217$

In $\mathrm{l}$ age I: Berzeifniz Der zum Dentiafer geere und zur Saijerliden

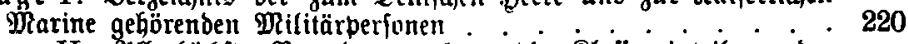

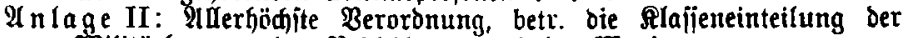
Milttärbeanten bes Reidgheeres uno der Marine . . . . . 221

II. A. Ariegsartikel für das fierr. . . . . . . . . 230

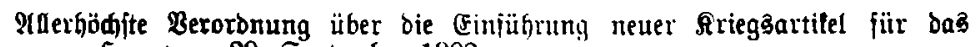
Seer vom 20. Eeptentber 1902 .......... 230 


\section{Ailitärftrafgeridtgord̀nung .}

\section{Erfter Teil: Berítederfafiung.}

Eriter Titel: Umfarig ber Militäritrajgetidt3barteit . . . . . . . . 260

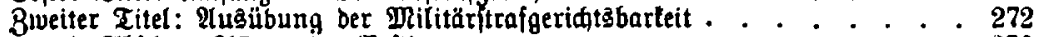

1. Ábidn.: Yagemeine Bejtimmungen . . . . . . . . 272

2. Ubjan.: Geridtobherr . . . . . . . . . . . . . . . 27

3. Abiфn.: Ertennende Geridte . . . . . . . . . . . . . . 291

I. Standgeridte . . . . . . . . . . . . 291

II. Sirieggeridte . . . . . . . . . . . . . . . . . 296

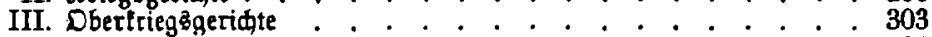

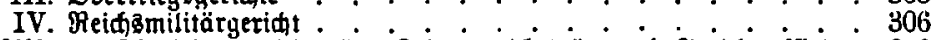

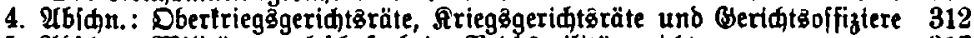

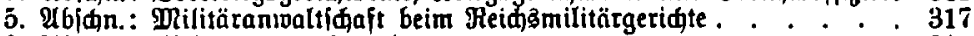

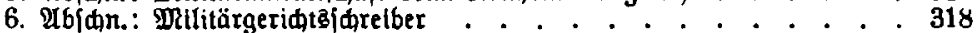

Dritter Titel: Militärjultizdertwaltung . . . . . . . . . . . . . . 319

\section{Biveiter Teil: Berfagren.}

Eriter Titel: Uldgemeine Beitimntungen . . . . . . . . . . . . . 322

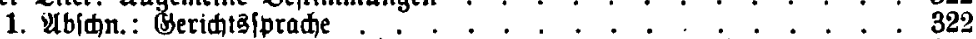

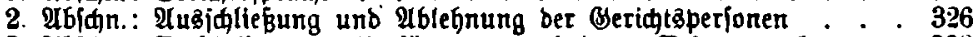

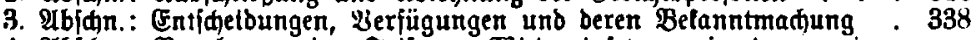

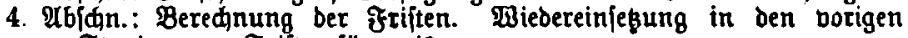
Stant gegen frijtoeriäumnis . . . . . . . . . . . . . 344

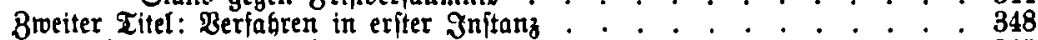

1. Ubjan.: Ermittelungsverjabren . . . . . . . . . . . . 348

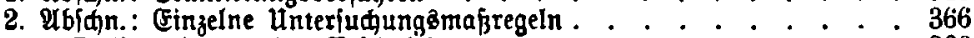

I. Wernehmung des Befiuldigten . . . . . . . 366

II. Einjtroeilige Enthebung Dom Dienjte. Berbaftung und vorläufige

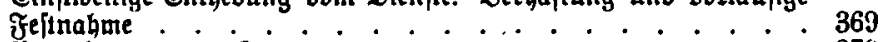

III. Bernehmung von Beugen . . . . . . . . . . . . . . 379

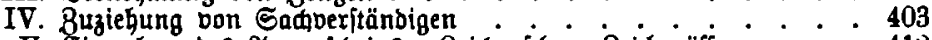

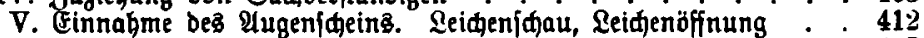

VI. Bejdlagnahme und Durdjudung ........ . 417

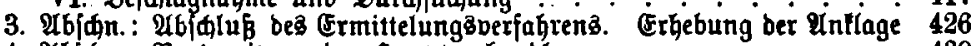

4. Ubidn.: Borbereitung ber fauptverfandlung . . . . . . . 439

5. 2bidn.: Gauptberhandlung . . . . . . . . . . 451

6. Ubfan.: : Berteibigung . . . . . . . . . . 528

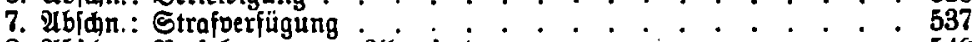

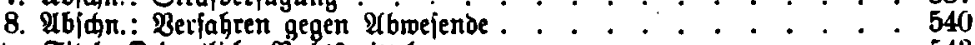

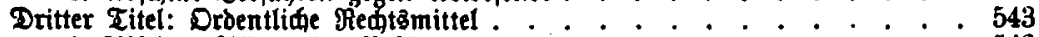

1. Ibian.: Angemeine Beftimmungen . . . . . . . . . . 543

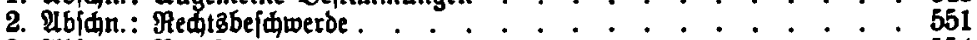

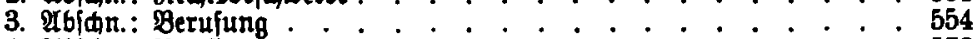

4. भbfan:: Revifion $: 572$

Bierter Ritel: Beftätigung ber im orbentlichen Berjabren ergangenen urteile 683

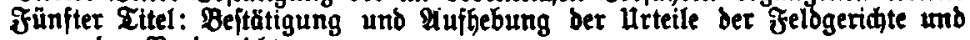
bet Borbgetidte ...................... 588 


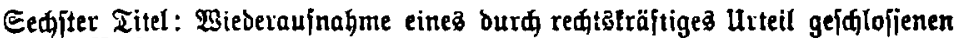
Berfabrena

Seite

Siebenter Zitel: Strajpolftrefung . . . . . . . . . .

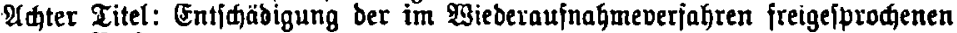
Berionen

Unlage: I: Berotonung, betr. das jtrafgeridutlide Berjahren gegen

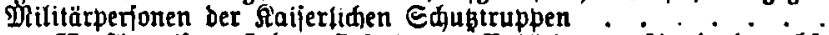

In lage II: 2Tnweifung I betr. Erjuchen ar Bebörben im Huslanbe nebjt Berzeidnia berjenigen fampeizerifđen Behörben, an bie unmittelbare Erfudjen um vorläufige ₹ejtnahme flühttger Berbreden geridtet werben tönnen . . . . . . . . . . . . . . . .

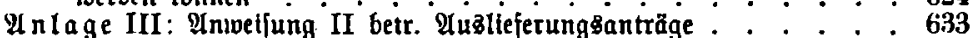

Anlage IV: Bebüftrenoronung für Beugen und Sadberitänbige. . 658

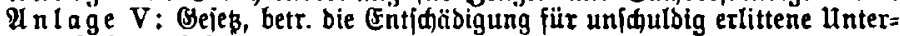
juhungåghaft . . . . . . . . . . . . 661

Anlage VI: Belez über den Belagerungozuftand . . . . . 663

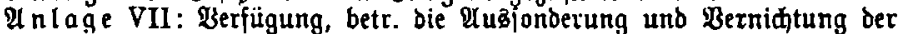

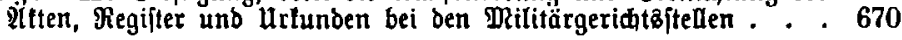

IV. A. Derordnang ïber die Ehrengeridte der (Offiziete im preufi [acn

ARere vom 2. Mai 1874 und Pllergid fite Drber bom 2. Nobember 187ó für ble $\mathfrak{M a r i n e}$

B. Derordnung über die Chrengeridte der Offiziere der \&ai (erliden

Alarine vơn 26. Jult 1895 und Ergänzung8orber vom 20. Deärz 1899

I. Zुwed ber Êhrengeridte

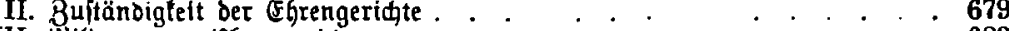

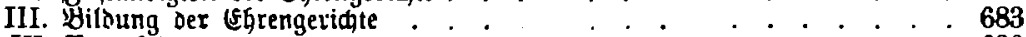

IV. Bom (Egtenrat . . . . . . . . . . . . . 690

V. Bon dem ebrengertittiden Berfafter . . . . . . . . . . . . 698

Beilage I. Beitimmungen über bie Bernefmung des Pingejđuldigten. 728

Beilage II. Paiter zur Borladung von Beugen ....... 728

Beilage III. Bejttmmungen übcr bie Bernehmung von Beugen burd Den Efrenrat. . . . . . . . . . .

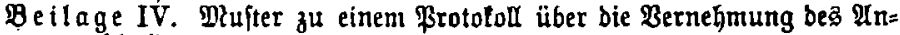
gejuulbigten . . . . . .

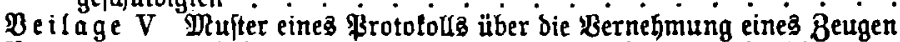

Betlage VI. Drufter zu einer eḩrengeridtlidjen Sprudverbanblung .

Beilage VII. Mamentlide Qijte ulm. . . . .

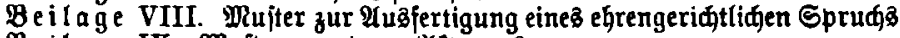

Beilage IX. Dufter zu einem Mltenasgzuge

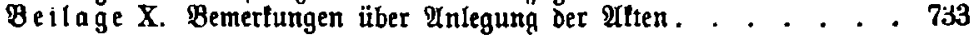

Il nlage I: Ergänzungsorber vom 1. Jaluar 1897 . . . . . 734

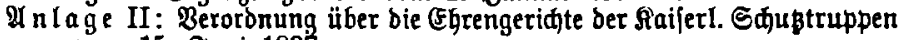
Dom 15. З̧นni 1897

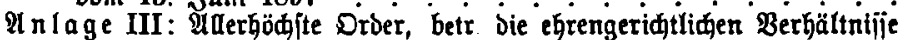

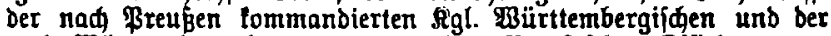

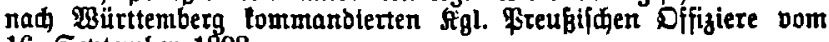
16. September 1898

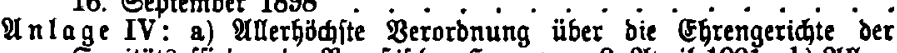

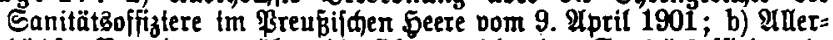

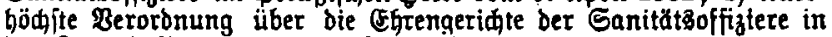
Der Raijerl. Mlarine pom 3. Junt 1901 .

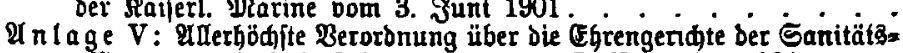
offiziere Der Siaiferl. Squatruppen bom 7. Noventber 1901 . . 
T. A Digziplinarftrafordnung für

1. Ubidn.: Umfang ber Diłziplinarjtrafgerwalt . . . . . . . . 763

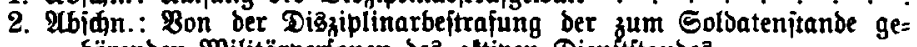

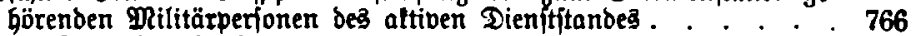

I. Dişziplinaritrafen . . . . . . . . . . . . . 766

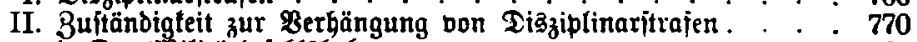

1. Der yhilitärbejęlăhaber 770

2. Der im Borgejeştenverbältnis itehenden Writglieder ber Sani= tätătorps

3. Abiđn.: Bon ber Disziblinarbeftrafung Der zum Soldatenitanide ge:

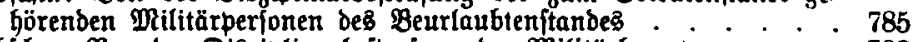

4. :

5. Abjan.: Bon ber Dišziplinarbejtrafung ber im $\$ 2$ unter $\mathfrak{M r} .3$ und $\dot{4}$ genannten \$erionen . . . . . . . . . .

6. Abian.: Bon ber Aubübung ber Diģiplinarjtrafgenalt uns von ber Bollitredung ber Disziplinarftrafen . . . . . . . . . . . 796

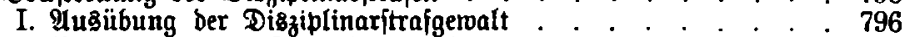

II. Bollitredung ber Diszjiplinarjtrafer . . . . . . . . . . $\quad .800$

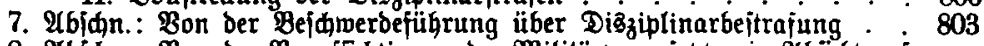

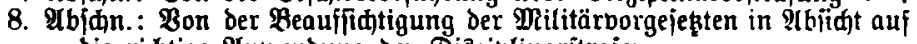
bie riatige Anmendung ber Diaziplinaritrajen. . . . . . 804

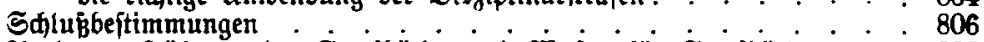

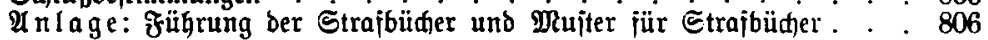

B. Disziplinarftrafordnang für die Rai(erlide Aarine vom 1. गio= vember 1902 . . . . . . . . . . . . . . . . . . . . 812

1. Mbidn.: Umjang ber Straigewalt . . . . . . . . . 813

2. थbjan.: A. Bejtrajung ber zum Solbatenjtande gebörenden Militär:

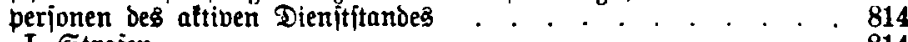

I. Strajen

II. Buftändigfeit . . . . . . . . . . . . . . . . . . 817

B. Bejtrafung ber ßerjonen Des Beurlaubtenftandes . . . . . 832

3. Abjun.: Beitrafung der $\mathfrak{D}$ ilititärbeamten der Marine . . . 835

4. Abjan.: Beftrafung der im \&2 unter ben Biffern 2 (an Bord), 3 uni 4 aufgefübrten und ber vertrng 3mäbig angejtellten ßerjonen jomie ber Rriegagefangenen . . . . . . . . . . 839

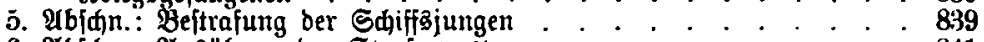

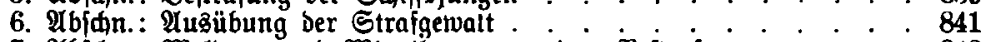

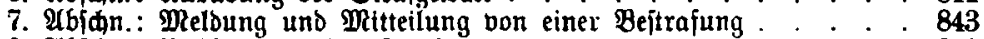

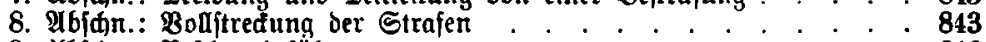

9. ) ubjan.: Bejthwerdeführung . . . . . . . . . . . . . . . 846

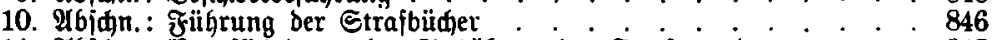

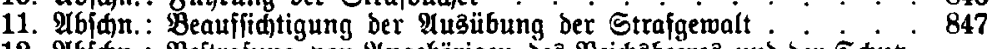

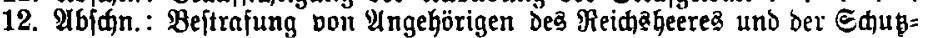
truppen . . . . . . . . . . . . . . . . 848

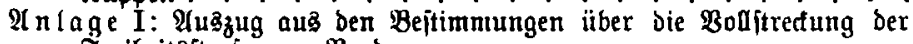
گreiheitşitrajen an Borb . . . . . . . . . . . . . . 848

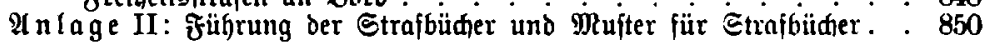

C. Ge[et, betr. Die Dienftuergehen der ridhterliden Antilitärjufi\}beamten and die unfreimillige Derfégung derfelben in eine andere Stelle oder ill den Ruljeftand vom 1. Dezember 1898 . 853 थXgemeine Bé́timmungen über Dienjtvergeben und deren Beftrajung . 853 Beriaffung und Buftänbigleit ber Dişziplinargeriđte . . . . . . . 856 Berfahren bei ben Dişjiplinargeridten . . . . . . . . . . . . 858 
Seite

Borläujige Dienjtenthebung . . . . . . . . . . . . . . . . 863

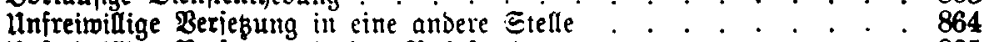

Unjreimillige Berjegung in ben Mufejtans. . . . . . . . . . . 865

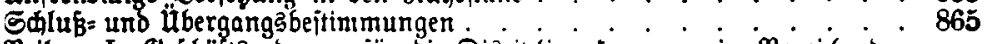

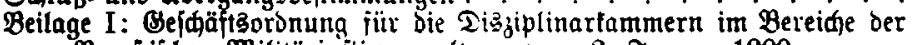

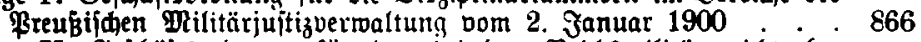

Beilage II: Bejđäjtsoronung fïr ben hei bem Reidsmilitärgeridite be= jtegenden Dišziplinargơ f̈̈r ridterlidie Militärjujtizbeamte . . . 869

VI. A. Bef中werdeardnang für dag fieer . . . . . . . . , 872

I. Bom 30. März 1895 . . . . . . . . . . . . . . . . . . . . . . 873

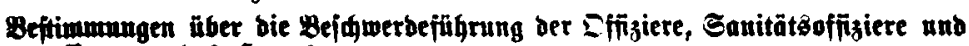

Deanten bes \$eeres

I. Offixiere unb Sanitäts̄offizicre. . . . . . . . . . . . 873

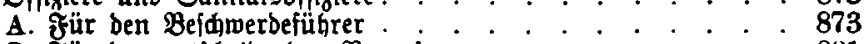

II. Beamte den entjđeidenden Borgejegten.

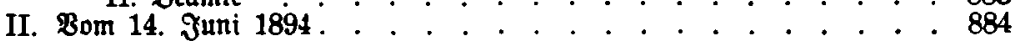

Beftimunngen aber bie Bejuwerbeführung ber Berionen bes Eolbatenitunbes

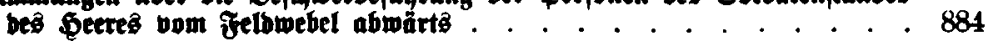

I. F̈ür ben Befdrerbefüfrer . . . . . . . . . . . . . . . 884

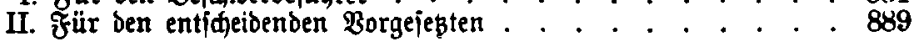

B. Bc/h)werdeordnang für die Aniferlide fatarine . . . . . 872

I. Bom 30. Deaember 1895 . . . . . . . . . . . . . . . . . 873

Beftimmungen über bie Bejdwerbeführung ber Dffïiere ber §aiferliden Dlarine,

fomie ber \$eanten ber Marineverwaltung. . . . . . . . 873

I. Offiziere . . . . . . . . . . . . . . . . . 873

A. Jü̈r ben Bejqmerbeführer . . . . . . . . . . . . 873

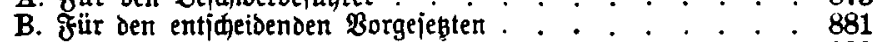

II. Beamte

II. Bom 23. Ottober 1894 . . . . . . . . . . . . . . . . . . . . 884

Beftimmungen über bie Bejđmerbefilgrung ber Berjouten bes Eolbatenitambes

ber \$aiferliqen Marine nom Defoffizier abuäts . . . . . . . . 884

I. Fïr ben Beiduerdefïgrer . . . . . . . . . . . . 884

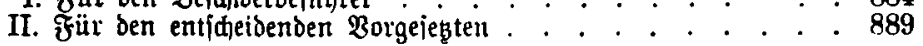

\section{Anbang.}

I. Strafge[egbud für Das Deut(d)e Krid) . . . . . . 895

A. Cinfübrungsgefes zum Strafgejębud bom 31. Mai 1870 . . . . 895

Meitere Einführungągejese

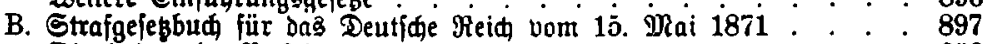

Finleitende Beftimungen

Eriter Teil: Bon ber Bejtrafung ber Berbreden, Bergegen und übertretungen im allgemeinen.

1. Ubidn.: Strafen . . . . . . . . . . . . . . . . 900

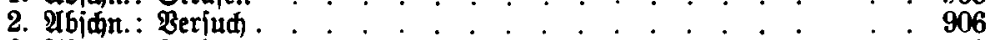

3. थbinn.: Tetlnahme 908

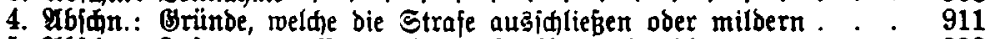

5. थbiфn.: Buiammentreffen mehrerer itrafbarer bandlungen. . . . 920 
3weiter Teil: Bon ben einzelnen Berbreden, Bergehen und übertretungen und beren Bejtrafung.

1. Ubiळn.: Sodperrat und నandegెverrat . . . . . . . . . . . 924

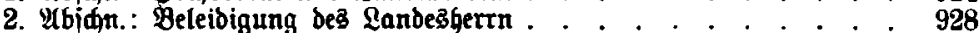

3. Ybian.: Beleibigung von Bunbesiüriten . . . . . . . . . . 929

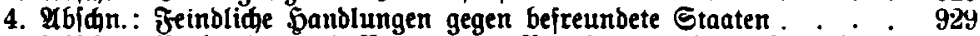

5. Mbidn.: Berbrewen und Bergeben in Beziegung auf bie Aus̈übung

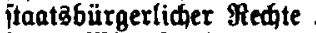

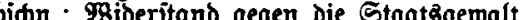

7. 9ufn: Betbrenen und

8. Ábidn.: פRünzberbreden und פzünzergehen

9. Mbidn.: gleineid . . . . . . . . . . . . . . . . . 945

10. Abjan.: falfae $\mathfrak{A n}\lceil$ dulbigung . . . . . . . . 950

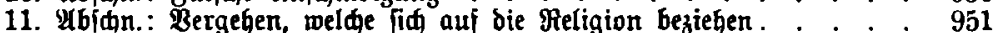

12. Ubidn.: Berbredien und Bergeben in Beziebung auf den \$erjonenitand 952

13. $\mathfrak{A b i d n . : ~ B e r b r e d e n ~ u n d ~ B e r g e b e n ~ m i b e r ~ b i e ~ S i t t l i d f e i t . ~ . ~ . ~ . ~ . ~} 952$

14. $\mathfrak{A b l d n . : ~ B e l e i b i g u n g ~ . ~ . ~ . ~ . ~ . ~ . ~ . ~ . ~ . ~ . ~ . ~ . ~ . ~ . ~ . ~ . ~ . ~} 958$

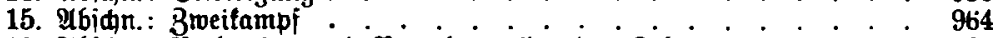

16. Mbiфn.: ßerbreden und Bergehen wiber baz Leben . . . . . . 965

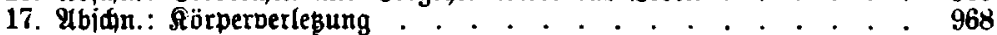

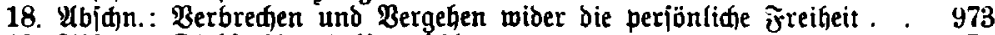

19. थbian.: Diebitahl und Unteriđlagung. . . . . 975

20. थbiqn.: Raub und Erpreijung . . . . . . . . . . . . . 981

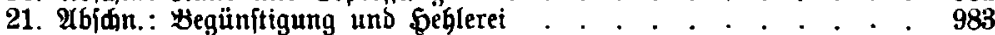

22. $\mathfrak{X b i q n . : ~ B e t r u g ~ u n d ~ u n t r e u e . ~ . ~ . ~ . ~ . ~ . ~ . ~ . ~ . ~ . ~ . ~} 986$

23. Xbidn.: Urtunbenfälidung . . . . . . . . . . . . . . . . . 988

24. Mbín,: Bantrott . . . . . . . . . . 992

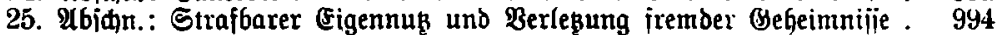

26. $\mathfrak{a b i q n . : ~ S a d b e j a j a ̈ b i g u n g ~ . ~ . ~ . ~ . ~ . ~ . ~ . ~ . ~ . ~ . ~ . ~ . ~ . ~} 998$

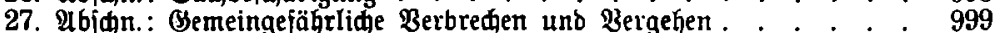

28. Abian.: Serbreden und Bergehen im Amte. . . . . . . . . 1003

29. $\mathfrak{A}$ bi由n.: Hubertretungen . . . . . . . . . . . . . . . . 1009

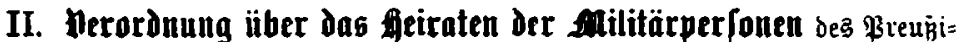
iden feeres uno der \$reußijacen \&andgendarmerie nebit den entipredsenden

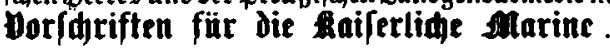

A. Seitaţెerfaubniz . . . . . . . . . . . . . . . . 1019

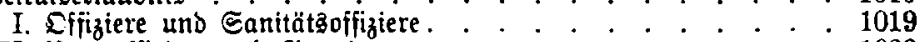

II. Unteroifiziere und Bemeine . . . . . . . . . . . . . . 1028

III. MRilitärbeamte . . . . . . . . . . . . . . . . . 1025

IV. Gemeinfdaftlide Beftimmungen . . . . . . . . . . . 1026

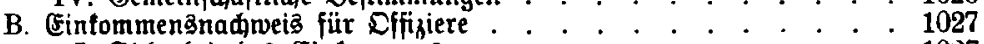

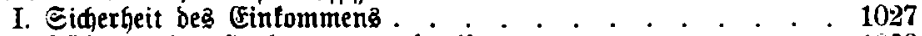

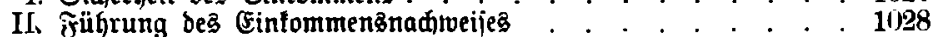

III. Einvilliqung ber Milttärbehörbe zu Şerfügungen itber das

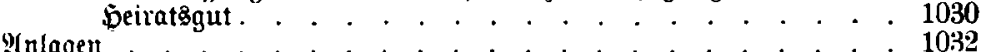

III. We (eb, betr. die freiwillige (Geridhtsbarkeit und andere hed)tgangelegenheiten in foer und sarine . . . . . . . . 1034

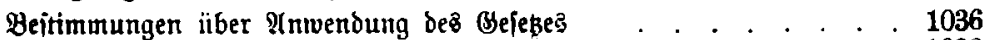

Mnlagen . . . . . . . . . . . . . . . . . . . . 1038

sadregifter . . . . . . . . . . . . . . . . . 1048 